\title{
Étude exploratoire de la pratique évaluative dans le domaine de la santé au Burkina Faso
}

\author{
Wamadini dite Minata Souratié \\ Université de Dédougou, Burkina Faso \\ Olivier G. Sossa \\ Université Thomas Sankara, Burkina Faso \\ Élisabeth Paul \\ Université Libre de Bruxelles, Belgique
}

\begin{abstract}
Résumé : Cette étude fournit une analyse réflexive de la pratique évaluative dans le domaine de la santé au Burkina Faso. Pour ce faire, une analyse d'un échantillon de dix évaluations réalisées dans le secteur de la santé depuis 2011 a été conduite. Il ressort de cette analyse descriptive que les pratiques évaluatives actuelles sont très variables dans le pays. La majorité des rapports dévaluation produits ne respectent pas les standards et normes internationaux en matière dévaluation, notamment pour ce qui concerne la définition du mandat et des questions évaluatives, la précision des critères dévaluation et la qualité de la méthodologie utilisée. Nous concluons que le développement d'une culture évaluative et un renforcement des capacités sont nécessaires pour une meilleure capitalisation des expériences et pour l'utilisation des données probantes dans la prise de décision.
\end{abstract}

Mots clés : Burkina Faso, évaluation, pratique évaluative, secteur de la santé

\begin{abstract}
This study provides a reflexive analysis of evaluative practice in the health sector in Burkina Faso. A sample of ten evaluations carried out in the health sector since 2011 was analyzed. The descriptive analysis shows that current evaluation practices vary very widely across the country. Most evaluation reports do not comply with international norms and standards in evaluation, in particular with regard to the definition of terms of reference and evaluation questions, the precision of evaluation criteria and the quality of the methodology. We conclude that a culture of evaluation must be developed and capacity must be built for better capitalization of experiences and for evidence-based decision-making.
\end{abstract}

Keywords: Burkina Faso, evaluation, evaluation practice, health sector

Dans les pays en développement, les systèmes de santé font face à de nombreux défis, comme des problèmes de financement, de gouvernance, de ressources

Correspondance à l'auteur : Wamadini dite Minata Souratié, Université de Dédougou, Dédougou, Burkina Faso; souratieminata@yahoo.fr 
humaines, d'approvisionnement, de prestation de soins et de gestion de l'information sanitaire. Pour les relever, le secteur de la santé bénéficie d'importants soutiens internationaux. Ainsi, depuis plusieurs décennies, de nombreux organismes de santé mondiale interviennent pour renforcer les capacités d'actions des acteurs de santé publique dans ces pays afin d'améliorer leur système de santé. Toutefois, l'efficacité de ces interventions reste encore à prouver (Khan et coll., 2016; Ramaswamy, Kallam, Kopic, Pujic et Owen, 2016). Pour s'assurer de la pertinence et de l'efficacité des interventions, leur mise en œuvre doit se fonder sur des données probantes, mais ce n'est pas le cas de la plupart des interventions de santé mondiale (Morra et Rist, 2009). Or, les financements de politiques, programmes ou projets de santé nécessitent l'utilisation de bases probantes comme socle pour évaluer ces interventions et mesurer les progrès réalisés en matière d'amélioration de la santé. Chaque contexte étant différent, il est essentiel que les bases probantes soient développées au niveau de chaque pays, voire au niveau de chaque région. Ainsi, des évaluations rigoureuses sont nécessaires pour déterminer si les interventions sanitaires sont performantes dans leur contexte.

L'évaluation est définie par l'Organisation de coopération et de développement économiques (OCDE) comme une « appréciation systématique et objective d'un projet, d'un programme ou d'une politique, en cours ou terminé, de sa conception, de sa mise en œuvre et de ses résultats " (OCDE, 2002). Lévaluation permet, d'une part, de rendre des comptes dans une perspective d'imputabilité (évaluation sommative) et, d'autre part, d'améliorer un programme à travers la production d'informations utiles pour corriger, au besoin, la manière dont les activités sont mises en œuvre (évaluation formative) (Ridde et Dagenais, 2012). Plusieurs critères sont utilisés dans la littérature pour évaluer les projets, programmes ou politiques. De nombreux bailleurs de fonds utilisent couramment ceux du comité d'aide au développement (CAD) de l'OCDE, qui définit cinq principaux critères soit la pertinence, l'efficacité, l'efficience, l'impact et la durabilité/pérennité (CAD, 1991). Tout récemment, un nouveau critère a été ajouté soit celui de la cohérence (Réseau du CAD de l'OCDE, 2019). D’autres critères sont définis dans la littérature (Widmer, Landert et Bachman, 2000; Groupe des Nations Unies pour l'évaluation, 2016), mais l'utilisation de ces critères doit être adaptée au contexte de l'intervention et aux besoins des utilisateurs potentiels de l'évaluation (Réseau du CAD de l'OCDE, 2019). Les évaluations devraient ainsi s'appuyer sur des questionnements bien précis afin d'aboutir à des réponses précises.

\section{BREF APERÇU DE LA PRATIQUE ÉVALUATIVE EN AFRIQUE}

En Afrique, les interventions publiques ne font pas systématiquement lobjet d'une évaluation. En effet, même si, depuis 1999, l'Association africaine d'évaluation (AfrEA) a vu le jour dans loptique de promouvoir et de renforcer la pratique évaluative en Afrique, dans la pratique, l'évaluation reste rare (Dixey et Njai, 2013). Même quand les interventions sont évaluées, ces évaluations sont très souvent imposées par les bailleurs de fonds externes et sont généralement réalisées par des consultants 
(nationaux comme internationaux) dont l'indépendance est sujette à caution (Olivier de Sardan, 2011, Lavigne-Delville, 2011, Lavigne-Delville, 2016).

Par ailleurs, les travaux réflexifs concernant les pratiques évaluatives en Afrique sont assez rares parce que pendant longtemps, il n'existait pas de revue africaine permettant de les valoriser (Ridde, Kouanda et Kobiané, 2016). Ce n'est quà la fin de l'année 2013, qu'a été lancé le premier numéro du Journal africain de l'évaluation. On déplore cependant le peu de publications d'évaluation issues de pays francophones.

La pratique évaluative en Afrique est confrontée à de nombreux enjeux, allant des aspects méthodologiques à l'utilisation des résultats. En effet, les problèmes méthodologiques se trouvent notamment au niveau du devis évaluatif, de la disponibilité des données de base et du choix de l'échantillon (Kouanda, 2016). Le problème qui se pose au niveau de la construction du devis évaluatif est que la décision d'évaluer n'est généralement pas prise avant la mise en œuvre du programme; pour ceux qui y pensent, ils précisent uniquement le type d'évaluation (évaluation à mi-parcours, évaluation finale, etc.) sans penser au cadre conceptuel d'un système évaluatif judicieux (Kouanda, 2016). Les devis robustes nécessitent la collecte des données avant la mise en œuvre de l'intervention. Lévaluation est tout un processus, depuis lélaboration du programme jusqu'à la fin de l'intervention en passant par sa mise en œuvre, et non une activité ponctuelle comme on peut le constater souvent dans la pratique. Ainsi, elle constitue un élément essentiel dans la mise en œuvre de toute intervention (Mimche, 2016).

Le choix de l'échantillonnage est également problématique. En effet, le choix des enquêtés est généralement guidé par les responsables de projet/programme parce que ces derniers perçoivent l'évaluation comme étant une procédure de contrôle de leur action (Kouanda, 2016). Par conséquent, un problème méthodologique lié à la validité des résultats se pose. D’un autre côté, la disponibilité des données est cruciale pour conduire une bonne évaluation. Cependant, la décision d'évaluer étant prise à la fin ou pendant l'exécution du programme fait qu'il n'y a pas d'informations sur la situation avant la mise en ouvre du programme (Kouanda, 2016).

Les évaluations devraient servir, à travers leur utilisation, à améliorer la mise en œuvre des interventions et leurs résultats. Mais la question de l'utilisation des résultats d'évaluation à des fins de prise de décision ne se pose souvent pas au moment de la restitution des résultats de ces évaluations (Ridde et coll., 2016). Ainsi, il existe un écart entre la réalisation des évaluations et leur utilisation dans la sphère de prise de décision politique. Cela peut se justifier par le fait que l'évaluation est méconnue par les acteurs. Elle est souvent perçue, non pas comme une évaluation du programme, mais comme un contrôle des premiers responsables dudit programme (Ridde et coll., 2016). Dans un tel contexte, les recommandations ne sont pas admises et les résultats restent inexploitables pour les prises de décisions.

\section{CONTEXTE DU BURKINA FASO}

Comme d'autres pays d'Afrique, au Burkina Faso, l'évaluation des politiques publiques n'est pas systématique et cette demande d'évaluer est essentiellement faite 
par les partenaires techniques et financiers. La pratique évaluative dans le pays est encore dans un état embryonnaire. En effet, un diagnostic des capacités évaluatives au Burkina Faso a identifié plusieurs faiblesses liées à la pratique évaluative dont : l'absence de cadre de référence et de cadres conceptuel et terminologique propre à l'évaluation de politique publique; l'absence d'une conception claire de ce qu'est l'évaluation; la faible qualité des rapports d'évaluation produits; le manque de coordination des pratiques et approches en matière de suivi et d'évaluation; et l'absence de dispositif pour l'évaluation des politiques publiques au niveau local (MINEFID/DEP, 2017). Il ressort de ce diagnostic que la méthodologie semble ne pas être maitrisée, puisque les rapports produits sont de nature plus descriptive qu'analytique. Une autre caractéristique de ces rapports d'évaluation est qu'ils sont orientés essentiellement sur la performance dans la réalisation des activités (efficacité, efficience). Toutefois, la performance est un concept multidimensionnel et non consensuel, mais souvent (dans la pratique) réduit à sa plus simple expression et parfois même confondu avec l'efficacité. Par exemple, l'OCDE (2002) définit la performance comme la «mesure selon laquelle l'action de développement, ou un partenaire, opère selon des critères, des normes, des orientations spécifiques, ou obtient des résultats conformes aux objectifs affichés ou planifiés ».

Au Burkina Faso, le plan national de développement sanitaire (PNDS) (20112020) relève plusieurs problèmes prioritaires auxquels est confronté le système national de santé. Ces problèmes portent principalement sur des questions de gouvernance et de leadership, notamment dans l'organisation et le fonctionnement des services de santé, la qualité des services et des soins de santé dans les formations sanitaires, les soins de santé communautaire, de la lutte contre le VIH/ SIDA, l'équité et l'accessibilité des services de santé (Ministère de la santé, 2011). Ces constats ont été corroborés plus récemment par la commission d'enquête parlementaire sur le système de santé au Burkina Faso (Assemblée nationale du Burkina Faso, 2017). Dans un tel contexte, l'évaluation s'avère être un outil indispensable pour soutenir l'adaptation des politiques nationales et des initiatives mondiales ainsi que l'organisation et le fonctionnement du système de santé, en vue d'en optimiser la performance. Cependant, au Burkina Faso, il n'existe pas jusquà présent de politique en matière d'évaluation ni au niveau global (Ministère de l'Economie, des Finances et du Développement, Direction de l'Economie et de la Planification [MINEFID/DEP], 2017) ni au ministère de la Santé. Ainsi, la décision d'évaluer ou non une intervention est souvent aléatoire et les évaluations sont souvent réalisées pour répondre aux exigences des bailleurs de fonds dans l’objectif de redevabilité des financements (Konkobo, 2013). Par conséquent, la capitalisation des résultats en vue de prise de décision reste problématique.

\section{OBJECTIFS}

Cette étude vise à réaliser une analyse exploratoire des pratiques évaluatives en santé au Burkina Faso en vue de les renforcer pour leur meilleure utilisation dans les prises de décision. Lobjectif de cet article est de faire un état des lieux critique des pratiques évaluatives dans le domaine de la santé au Burkina Faso. 
Cette recherche s'inscrit dans le cadre d'un projet de coopération universitaire qui vise à appuyer l'institutionnalisation de la culture évaluative dans le secteur de la santé au Burkina Faso, notamment en menant une méta-évaluation d'un échantillon dévaluations représentatives du secteur. Avant d’en arriver là, il fallait débroussailler le terrain en identifiant les principaux acteurs ainsi que les grandes tendances de la pratique évaluative dans le secteur, y compris les forces et les faiblesses des évaluations actuellement disponibles. Cette recherche constitue la première étape de ce projet. À cette fin, nous avons procédé à une première collecte de données, à une sélection des évaluations les plus pertinentes, puis à une analyse des caractéristiques clés des évaluations identifiées. Ensuite, il s’en est suivi une synthèse des grandes tendances de la pratique évaluative actuelle des politiques de santé au Burkina Faso.

\section{MÉTHODOLOGIE}

Cette recherche a consisté en une analyse descriptive d'un échantillon dévaluations en santé au Burkina Faso. Pour ce faire, plusieurs stratégies ont été utilisées pour arriver au choix final des évaluations retenues dans le cadre de cette étude.

Nous avons d'abord procédé à une collecte des évaluations réalisées dans le secteur de la santé au Burkina Faso, quelle que soit leur source de financement ou quel que soit l'organisme chargé de l'évaluation, menées à terme depuis le début de la mise en œuvre du PNDS 2011-2020. La collecte de ces évaluations a été possible avec l'appui de l'unité de gestion et transfert des connaissances et des services responsables du suivi-évaluation des projets/programmes du Ministère de la santé du Burkina Faso. Elle a également été réalisée grâce à l'exploration de sites Web des principaux organismes internationaux qui financent les projets et programmes relatifs à la santé dans le pays, ainsi que des contacts individuels qui nous ont permis dobtenir des publications des chercheurs qui travaillent sur les questions relatives à la santé. Au total, 22 rapports ont été identifiés et ce nombre nest probablement pas exhaustif.

Parmi les rapports collectés, nous avons sélectionné les rapports qui consistaient en de "réelles évaluations ", à entendre par là, des rapports ayant une visée analytique, dont les objectifs et/ou questions dévaluation étaient définis, et qui utilisaient une méthodologie spécifiquement conçue pour répondre à ces objectifs. En outre, deux revues de performances de programmes ont été retenues dans léchantillon du fait qu'elles utilisaient de l'information systématique pour poser un jugement sur la valeur des programmes. Il faut remarquer que certains rapports initialement collectés se sont révélés être de simples rapports d'enquête n’ayant pas de visée analytique, mais présentant simplement les résultats en matière de prévalence de certaines maladies. Une fois nos critères d'inclusion appliqués, dix rapports d'évaluation ont été retenus pour l'analyse (tableau 1).

Outre les critères d'inclusion de notre échantillon à analyser, nous avons développé notre propre cadre d'analyse sur la base d'une revue de littérature sur les critères de méta-évaluation. Nous avons choisi les critères qui nous semblaient les plus pertinents pour asseoir la suite de notre projet et avons notamment retenu 


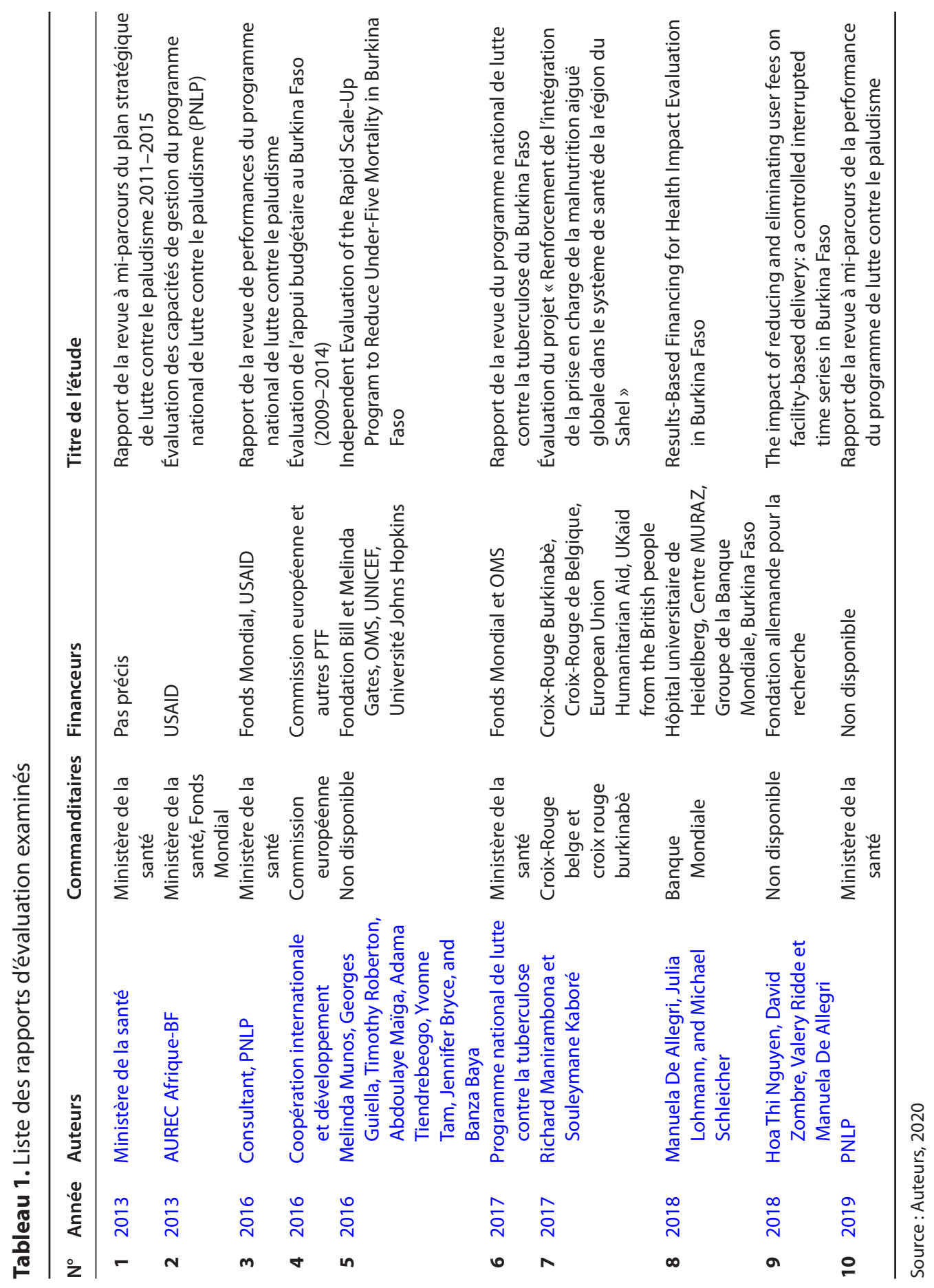


les critères d'utilité, de faisabilité, de crédibilité (Scriven, 2011; Daigneault, 2010; Cooksy et Caracelli, 2009; Widmer et coll., 2000). Ainsi, notre cadre repose sur plusieurs critères : les critères de satisfaction de la demande, de justification de la méthode, de fiabilité des données, de solidité de l'analyse, de crédibilité des constats, d'utilité des recommandations et de clarté des rapports.

Notons d’emblée que notre collecte de données n’a pas permis de récolter que des rapports finaux et publiés des évaluations concernées, mais que nous n’avons pas été en mesure, à ce stade, de récolter des documents complémentaires. Notre analyse s'est donc penchée uniquement sur les rapports d'évaluations validés.

Sur la base des critères retenus, l'examen des rapports d'évaluation de notre échantillon a permis d'identifier leurs principales caractéristiques qui nous ont permis d'apprécier la qualité de la pratique évaluative actuelle dans le domaine de la santé au Burkina Faso. Le tableau 2 présente les différents critères retenus dans cette recherche ainsi que leur définition.

Tableau 2. Définition des critères

\begin{tabular}{|c|c|}
\hline Critères & Définitions \\
\hline $\begin{array}{l}\text { Satisfaction de la } \\
\text { demande }\end{array}$ & $\begin{array}{l}\text { Les questions de l'évaluation abordées sont-elles conformes } \\
\text { aux termes de référence? }\end{array}$ \\
\hline $\begin{array}{l}\text { Justification de la } \\
\text { méthode }\end{array}$ & $\begin{array}{l}\text { Le rapport comprend-il une critique de la méthode } \\
\text { d'évaluation et des choix méthodologiques? Les limites de } \\
\text { la méthodologie sont-elles présentées? }\end{array}$ \\
\hline $\begin{array}{c}\text { Fiabilité des } \\
\text { données }\end{array}$ & $\begin{array}{l}\text { Les biais pouvant émaner des informations collectées sont-ils } \\
\text { analysés? Des stratégies visant à réduire ces biais ont-elles } \\
\text { été proposées et utilisées? }\end{array}$ \\
\hline Solidité de l'analyse & $\begin{array}{l}\text { Les biais en lien avec la stratégie d'analyse sont-ils discutés, } \\
\text { ainsi que leurs conséquences sur la validité de l'analyse? }\end{array}$ \\
\hline $\begin{array}{l}\text { Crédibilité des } \\
\text { constats }\end{array}$ & $\begin{array}{l}\text { Les facteurs contextuels et leur influence sur l'intervention } \\
\text { évaluée (ainsi que la démarche évaluative) ont-ils été } \\
\text { mis en évidence? Les biais dans le choix des hypothèses } \\
\text { interprétatives et les extrapolations faites sont-ils analysés } \\
\text { et leurs conséquences explicitées? }\end{array}$ \\
\hline $\begin{array}{l}\text { Validité des } \\
\text { conclusions }\end{array}$ & $\begin{array}{l}\text { Les conclusions sont-elles basées sur l'analyse des constats? } \\
\text { Sont-elles présentées selon leur importance, en rapport } \\
\text { avec la globalité de l'intervention évaluée et prennent-elles } \\
\text { en compte les relations entre le contexte et l'intervention? }\end{array}$ \\
\hline $\begin{array}{l}\text { Utilité des } \\
\text { recommandations }\end{array}$ & $\begin{array}{l}\text { Les recommandations sont-elles hiérarchisées et } \\
\text { opérationnalisables? }\end{array}$ \\
\hline Clarté des rapports & $\begin{array}{l}\text { Le rapport est-il bien structuré, le résumé est-il en lui seul } \\
\text { opérationnel? }\end{array}$ \\
\hline
\end{tabular}

Source : Auteurs sur base de la revue de littérature (Service de l'Évaluation spéciale, 2013; Scriven, 2011; Daigneault, 2010; Cooksy et Caracelli, 2009; Stufflebeam, 2001;Widmer et coll., 2000; Conseil scientifique de l'évaluation, 1996) 


\section{CARACTÉRISTIQUES DE L'ÉCHANTILLON DES RAPPORTS D'ÉVALUATION EXAMINÉS}

Cette section présente les résultats de l'examen de notre échantillon de rapports d'évaluation. Elle analyse dans un premier point les principales caractéristiques des rapports étudiés et présente dans un second point la méthodologie adoptée lors de ces évaluations. Le tableau 2 résume les principales caractéristiques des rapports d'évaluation retenus.

\section{Principales caractéristiques des rapports d'évaluation}

Nous avons examiné un échantillon non exhaustif de dix rapports dévaluation réalisés dans le secteur de la santé du Burkina Faso depuis 2011. Ces évaluations ont porté sur toute une série de sujets : le paludisme $(n=4)$, la tuberculose $(n=1)$, la malnutrition des enfants $(n=1)$, le financement basé sur les résultats (abordant principalement les questions de santé maternelle et infantile $[n=1])$, la santé maternelle $(n=1)$, la mortalité des enfants de moins de 5 ans $(n=1)$ et l'appui budgétaire au Burkina Faso (dont les questions relatives à la santé portent sur les trois principales thématiques que sont la santé maternelle, l'état nutritionnel des enfants de moins de 5 ans et les maladies endémo-épidémiques telles que le paludisme, VIH/SIDA et rougeole $[n=1]$ ). Parmi ces dix rapports examinés, deux sont des revues de performance, dont l'une porte sur l'évaluation des capacités de gestion du programme national de lutte contre le paludisme (PNLP) et l'autre sur la performance du programme national de lutte contre la tuberculose (PNLT). Dans la suite de nos analyses, les numéros des rapports correspondent à l'ordre des numéros dans le tableau 1.

L'examen des rapports de notre échantillon montre que les commanditaires des évaluations ne sont pas expressément précisés dans la majorité des cas. On peut identifier les commanditaires dans seulement trois rapports $\left(n^{\circ} 4,7\right.$ et 8$)$. Toutefois, les évaluations examinées sont quasiment toutes (excepté les évaluations $\mathrm{n}^{\circ} 1$ et 10 qui ne précisent pas les bailleurs de fonds) financées par les organismes non gouvernementaux et institutions internationales, notamment l'Agence des États-Unis pour le développement international (USAID), le Fonds Mondial de lutte contre le sida, la tuberculose et le paludisme, l'Organisation mondiale de la santé (OMS), le Fonds des Nations-Unies pour l'Enfance (UNICEF), la Fondation Bill et Melinda Gates, les sociétés nationales de la Croix-Rouge belge et burkinabè.

La quasi-totalité des rapports n'inclut pas les termes de références (TDR) de l'évaluation. En effet, les TDR sont présents à l'annexe des rapports dans seulement deux rapports d'évaluation ( $\mathrm{n}^{\circ} 4$ et 7$)$. Dans quatre rapports d'évaluations $\left(\mathrm{n}^{\circ} 1\right.$, 3, 6 et 10), les TDR sur lesquels devraient se baser les évaluateurs pour produire les évaluations répondant à la demande des commanditaires ne sont pas définis au préalable, mais plutôt élaborés lors de la première phase de l'évaluation (phase de la planification), au cours de laquelle l'équipe de coordination est constituée, les ressources financières sont mobilisées, les personnes-ressources externes sont identifiées et le(s) consultant(s) est (sont) identifié(s). Cependant, dans le rapport $\mathrm{n}^{\mathrm{o}} 10$, aucune information n'est donnée quant à la composition de l'équipe de 
coordination ainsi que les personnes-ressources externes identifiées. Toutefois, notons que certaines évaluations ont connu une dimension participative. En effet, il est mentionné dans les rapports $\left(\mathrm{n}^{\circ} 1,3,6\right.$ et 10) la participation de plusieurs partenaires depuis la validation des TDR jusquà la validation des résultats d'évaluation. Bien qu'il soit mentionné dans les rapports que les TDR aient été élaborés lors de la première phase de l'évaluation, ils restent cependant absents des rapports d'évaluation. Dans un seul rapport ( $\mathrm{n}^{\circ} 2$ ), le responsable du cabinet chargé de l'évaluation a juste fait mention du respect des TDR dans sa lettre adressée au coordonnateur du PNLP. Quant aux trois autres rapports d'évaluation ( $n^{\circ} 5,8$ et 9), réalisés par des chercheurs dont deux ( $n^{\circ} 5$ et 9 ) publiés dans des revues scientifiques, aucune précision n'est donnée s'il y a eu ou non des TDR (ou un protocole).

Bien que l'objectif des évaluations soit donné au démarrage de l'évaluation, il n’en est pas de même pour les questions évaluatives. En général, ces questions ne sont pas explicitement posées dès le début de l'évaluation. L'analyse de notre échantillon d'évaluations montre que seules les trois évaluations dont les commanditaires sont explicitement mentionnés dans les rapports comportent des questions d'évaluation. Cependant, les questions posées dans le rapport $n^{\circ} 8$ portent uniquement sur l'efficacité du programme. Par exemple, les questions dans ledit rapport sont formulées de la sorte : " quel est l'effet de l'intervention sur des indicateurs? ». En outre, les questions évaluatives posées dans le rapport $n^{\circ} 7$, une évaluation commanditée par les Croix-Rouge belge et burkinabè et réalisée par deux consultants, sont des questions bien ciblées et correspondent aux critères retenus pour l'évaluation, lesquels critères ont été clairement définis. C’est d’ailleurs l'unique évaluation de l'échantillon qui a clairement lié les questions évaluatives avec les objectifs de l'évaluation basés sur les critères d'évaluation retenus.

Plus de la moitié des évaluations de l'échantillon (six) sont des évaluations ex-post qui sont par ailleurs des évaluations récapitulatives conduites à la fin du programme pour voir dans quelle mesure les objectifs escomptés ont été atteints (OCDE, 2002). Deux évaluations sont réalisées sur des programmes à mi-parcours $\left(n^{\circ} 1\right.$ et 10$)$ et deux autres ( $n^{\circ} 2$ et 6$)$ portent sur la performance des programmes (évaluations in itinere). Ces dernières (évaluations à mi-parcours et in itinere) sont de nature formative. Quant à l’objet évalué, la majorité des évaluations (huit) porte sur le processus et les résultats. Seulement une évaluation $\left(\mathrm{n}^{\circ} 2\right)$ porte uniquement sur le processus et une autre $\left(n^{\circ} 8\right)$ exclusivement sur les résultats.

Les critères sur lesquels repose l'évaluation ont été précisés dans un seul rapport d'évaluation de l'échantillon ( $\left.\mathrm{n}^{\circ} 7\right)$. Dans ce dernier, les critères d'évaluation retenus sont les critères de pertinence, d'efficacité, d'efficience, d'impact et de durabilité/pérennité. Toutefois, on peut observer dans l'examen des rapports d'évaluation que la majorité d'entre eux (excepté les deux revues de performance de programmes) traite implicitement de l'efficacité des interventions en mesurant particulièrement le niveau atteint des indicateurs. Également, le critère de pertinence est abordé dans la quasi-totalité des évaluations (dans neuf rapports), même si cela n'est pas explicitement mentionné, on peut le percevoir dans la présentation du contexte de mise en œuvre de la politique. Pour sa part, le critère d'efficience est abordé dans trois rapports d'évaluation, dont deux sont des évaluations à 
mi-parcours du PNLP ( $\mathrm{n}^{\circ} 1$ et 10 ) et une concerne l'évaluation sur la « malnutrition aiguë des enfants » (rapport $\mathrm{n}^{\circ} 7$ ). Cependant, le critère d'efficience n'est souvent pas bien compris par les évaluateurs. Il est apprécié dans deux évaluations ( $n^{\circ} 1$ et 10 ) sur la base du rapport entre le coût réel et le coût prévisionnel (alloué) en omettant que toutes les activités prévues à cet effet n’ont pas été mises en œuvre. Enfin, aucun rapport n'est établi entre les ressources mises en œuvre et les effets de l'intervention, ou encore entre les coûts de différentes options pour les mêmes résultats atteints par l'intervention.

\section{Démarche méthodologique des rapports d'évaluation}

L'analyse des rapports d'évaluation de notre échantillon montre que les évaluateurs ont utilisé différents outils de collecte et d'analyse des données, notamment les analyses documentaires, les entretiens, les enquêtes de terrain (données quantitatives), les analyses statistiques et/ou économétriques pour évaluer les politiques, programmes ou projets de santé au Burkina Faso, sans toutefois justifier le choix des différentes approches. En effet, le choix de la méthode utilisée n'est justifié que dans un seul rapport de l'échantillon ( $\left.n^{\circ} 8\right)$.

Dans trois rapports d'évaluation $\left(\mathrm{n}^{\circ} 1,3\right.$ et 10$)$, l'analyse documentaire, les entretiens et les analyses statistiques sont utilisés. Il s'agit des évaluations du PNLP qui sont essentiellement des évaluations internes, c'est-à-dire menées par les acteurs internes du programme (une seule a connu la participation d'un consultant dans l'équipe d'évaluateurs).

Dans quatre autres rapports d'évaluations ( $n^{\circ} 4,5,7$ et 8 ), les sources des données ont été précisées et le processus d'échantillonnage a été décrit. En effet, lévaluation du projet du financement basé sur les résultats $\left(\mathrm{n}^{\circ} 8\right)$ a utilisé une méthode quasi expérimentale avec une composante expérimentale imbriquée, c'est-à-dire que les districts d'intervention ont été choisis à dessein et les formations sanitaires ont été randomisées en des modalités d'intervention différentes. Les auteurs de l'évaluation ont fourni les informations sur les outils de collecte et d'analyse des données. Un système de contrôle des données a été mis en place pour s'assurer de la qualité des données collectées. Des outils économétriques (méthode de la double différence) ont été utilisés pour évaluer l'impact de l'intervention. Les limites de la méthodologie ont été discutées. Des stratégies ont été développées par les évaluateurs pour corriger les biais d'attribution et de problèmes liés à la taille de léchantillon. Par ailleurs, d'autres sources de données notamment les données du système national d'information sanitaire ont été utilisées pour vérifier la robustesse des résultats obtenus. Les interprétations des résultats sont faites à la lumière du contexte de mise en œuvre de l'intervention, des défis de mise en œuvre et des défis méthodologiques. Même si les auteurs n'ont pas pu isoler l'effet net du seul projet, ils ont tenté d'analyser les différentes situations qui pourraient conduire aux résultats observés avant de conclure qu'il serait nécessaire de conduire d'autres recherches afin de déterminer les effets nets de l'intervention (De Allegri, Lohmann et Schleicher, 2018).

En ce qui concerne le rapport $\mathrm{n}^{\circ} 7$ portant sur lévaluation sur la malnutrition aiguë des enfants, les évaluateurs ont eu recours à l'analyse documentaire, 
les entretiens approfondis, les groupes de discussion et les analyses statistiques afin de comparer les résultats des districts sanitaires. La procédure du choix de léchantillon a été décrite et l'équipe dévaluateurs a mis en œuvre un système de vérification des sources d'information afin de s'assurer de leur qualité. Pour l'analyse des données, les évaluateurs ont utilisé une matrice dévaluation qui permet de classer les différents critères dévaluation et de relier les questions dévaluation aux résultats obtenus. Des outils et tests statistiques ont été utilisés pour analyser l'association entre les variables. Le projet évalué s'inscrivant dans le cadre d'un programme débuté à l'avance, il nétait pas exclu que les effets observés puissent être causés par les actions des phases précédentes. Pour ainsi prendre en compte ces aspects dans l'analyse des différents effets observés, les évaluateurs ont utilisé plusieurs sources d'informations. Par ailleurs, des facteurs contextuels (à savoir l'implication de l'État, des organisations non gouvernementales, la prise de conscience de la population et même d'autres actions antérieures de la CroixRouge) ont été identifiés comme pouvant influencer les résultats du projet évalué (Manirambona et Kaboré, 2017).

Lévaluation indépendante du programme « Rapid Scale-Up » sur la réduction de la mortalité des enfants de moins de cinq ans (rapport $\mathrm{n}^{\circ}{ }^{5}$ ) s'est basée sur des analyses documentaires, des entretiens, des observations directes sur le terrain. Une description du processus de mise en œuvre du programme a permis le choix de la méthode dévaluation par les évaluateurs. Aussi, le processus déchantillonnage a été décrit et un système de suivi et de vérification des données a été mis en place pour contrôler la qualité des données collectées. Lors de lanalyse des données, on a utilisé une approche quasi expérimentale avec des analyses avant et après le programme, et la méthode de la double différence a été utilisée pour évaluer l'impact du programme. Les résultats ont été discutés au regard des facteurs contextuels. De cette analyse, l'équipe de recherche souligne la possibilité d'une faible influence des facteurs contextuels sur les résultats observés et tire ainsi la conclusion que les résultats peuvent donc être attribués au programme (Munos et coll., 2016).

Le rapport $\mathrm{n}^{\circ} 4$ qui a porté sur lévaluation de l'appui budgétaire au Burkina Faso et qui comporte des analyses sectorielles notamment relatives à la santé a utilisé un ensemble d’outils de collecte et d'analyse des données. Pour la collecte des données, les évaluateurs ont eu recours à la recherche documentaire, la compilation de base de données quantitatives, des entretiens individuels, des groupes de discussion, une enquête en ligne, des observations directes et des entretiens au niveau local. Quant à l'analyse des données, elle s’est appuyée sur des analyses de corrélation et les régressions à effets fixes. Les informations collectées ont été soumises à un processus d'analyse et de validation (par clustering, comparaison et triangulation). Les différentes sources d'information ont été croisées pour vérifier et affiner la description des mécanismes et des liens de causalité. Les auteurs ont discuté des défis liés à la fiabilité des données et ont conclu que ces défis n’entament pas la solidité des constatations observées (Coopération internationale et développement EuropeAid, 2016). 
Deux évaluations, à savoir les revues de performance des PNLP et PNLT, ont utilisé uniquement des méthodes qualitatives à travers des analyses documentaires et des entretiens pour identifier les forces et les faiblesses des différents programmes. Une seule évaluation de l'échantillon (rapport $n^{\circ} 9$ ) a fait recours uniquement à une méthode quantitative. Les évaluateurs ont utilisé des données quantitatives avec une approche quasi expérimentale utilisant une série chronologique interrompue contrôlée pour évaluer l'impact de l'intervention. Les résultats obtenus de ces estimations ont été analysés à la lumière des facteurs contextuels. Les auteurs soulignent une influence de ces facteurs contextuels sur les résultats observés sans toutefois pouvoir isoler l'effet net de l'intervention. Ainsi, les auteurs pensent qu'il faut des évaluations complémentaires pour expliquer certains résultats observés.

\section{DISCUSSION}

Cette étude exploratoire a permis de révéler les principales caractéristiques de la pratique évaluative actuelle dans le domaine de la santé au Burkina Faso. Il ressort de l'examen de notre échantillon de rapports d'évaluation que la pratique évaluative est très diversifiée. Il faut noter que l'absence des TDR dans les rapports ne permet pas de les apprécier par rapport aux attentes des commanditaires. Dès lors, nous nous sommes contentés uniquement de l'analyse des informations contenues dans les rapports, tout en les appréciant au regard du cadre d'analyse que nous avons développé sur la base de la revue de littérature.

La composition de l'équipe d'évaluateur n'est pas précisée dans certains rapports. Cette précision permettrait de connaître non seulement la compétence des évaluateurs à conduire l'évaluation, mais aussi leur indépendance par rapport aux organismes de mise en œuvre des interventions. L'indépendance, même si elle n'est pratiquement pas possible (Dubois, 2015), est souvent considérée comme l'un des critères de crédibilité des évaluations dans la littérature (Demarteau, 1998; OCDE, 2002; Dubois, 2015). Il faut toutefois noter que l'indépendance est un critère important dans la perspective de reddition des comptes, mais qui ne préjuge en rien de la qualité de l'évaluation et peut même parfois réduire la perspective d'appropriation et d'apprentissage.

Les critères d'évaluation ainsi que les questions évaluatives sont quasi absents des rapports d'évaluations examinés. Pourtant, ils constituent le socle de toute évaluation. À chaque critère d'évaluation devraient correspondre une ou plusieurs questions évaluatives traduisant les besoins des commanditaires, afin de permettre aux évaluateurs d’orienter leurs travaux vers les enjeux les plus importants (KPMG et Quadrant conseil, 2017). La définition des critères permet de mesurer les performances de l'objet évalué et ainsi de porter un jugement à travers les indicateurs obtenus. Les questions évaluatives permettent de mieux décrire les objectifs de l'évaluation - qui sont des déterminants de sa crédibilité et de son utilité - et d'orienter l'attention des évaluateurs vers les questions pertinentes pour la prise de décision - ce qui détermine l'utilité de l'évaluation. 


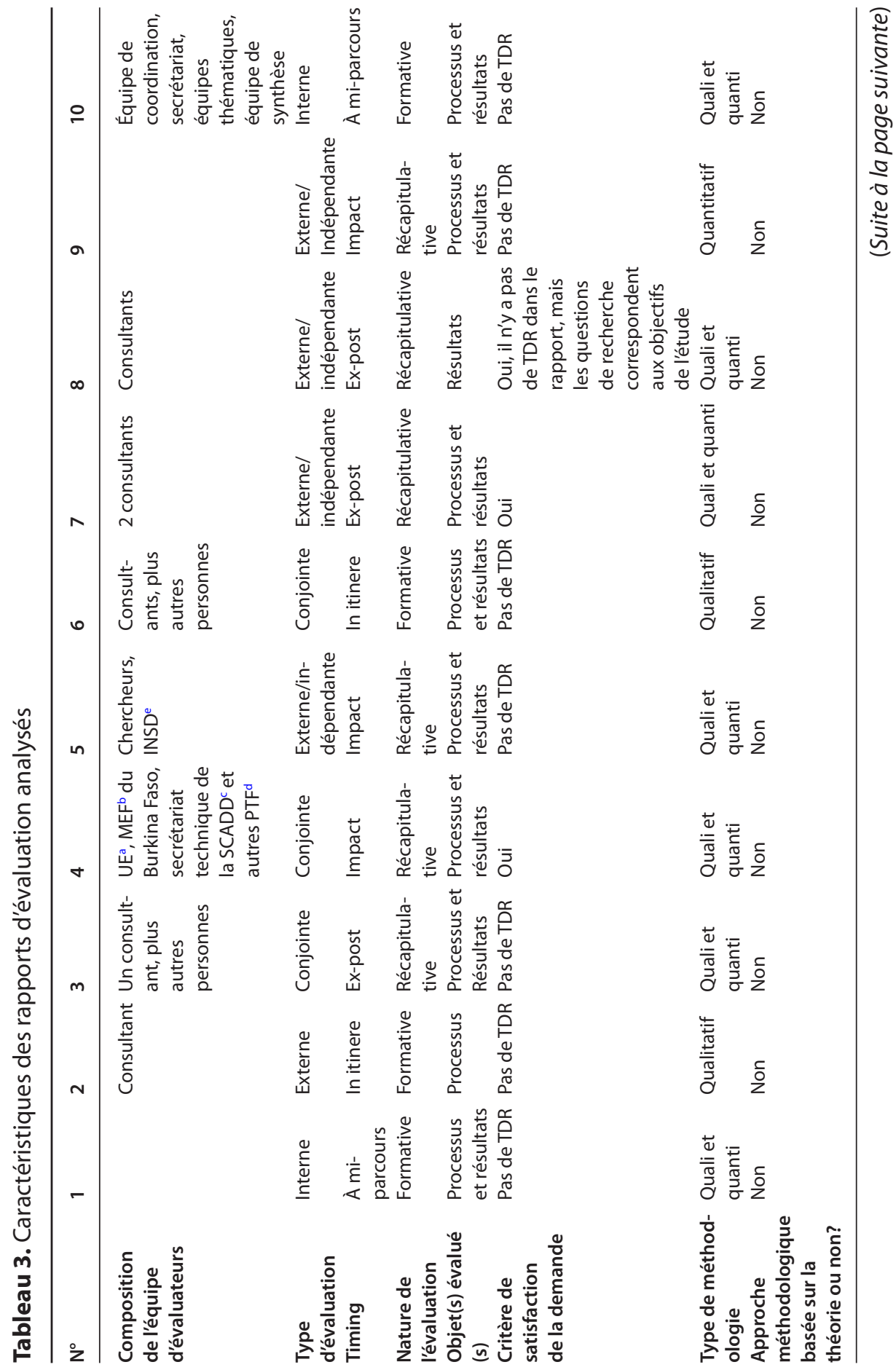




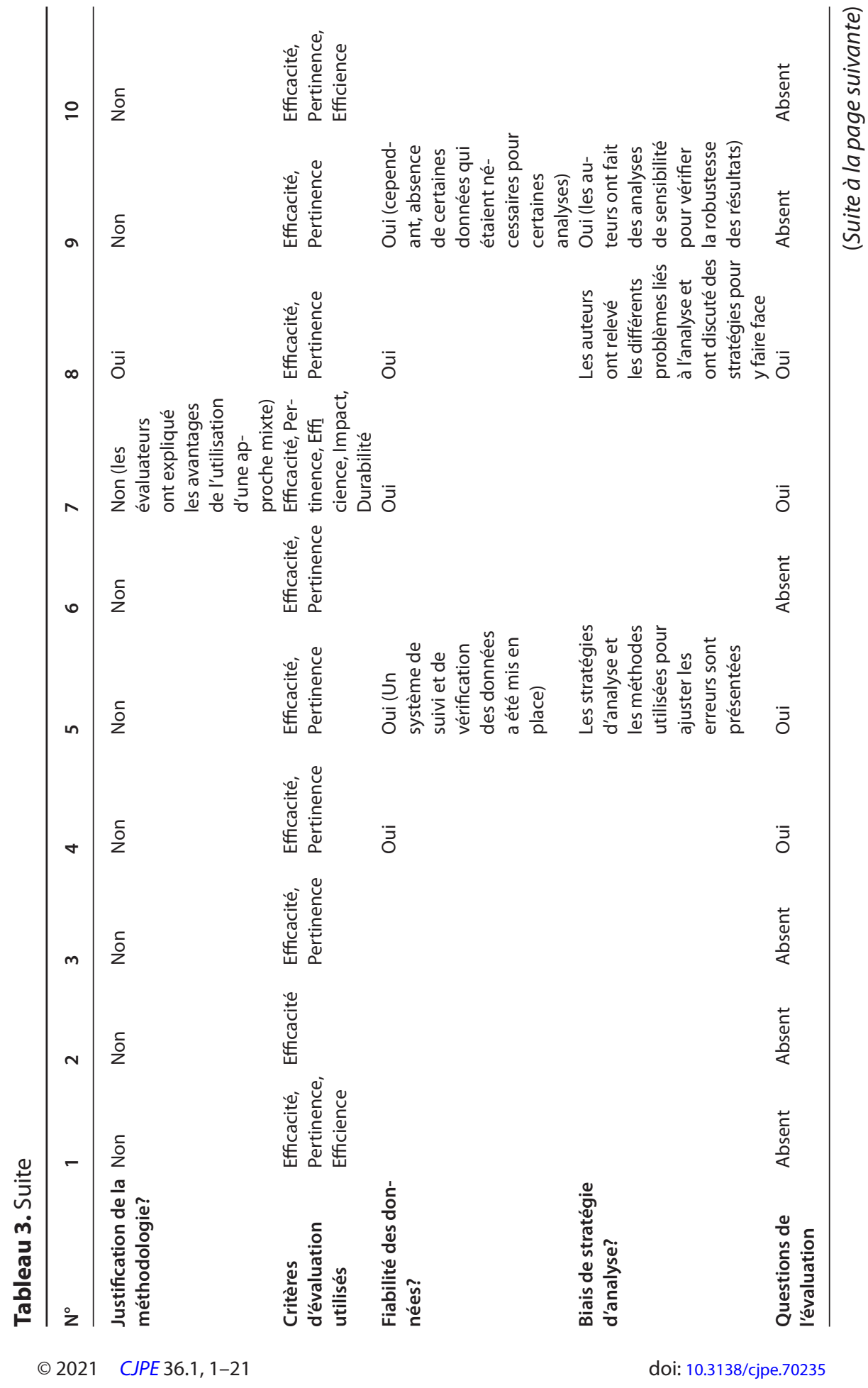




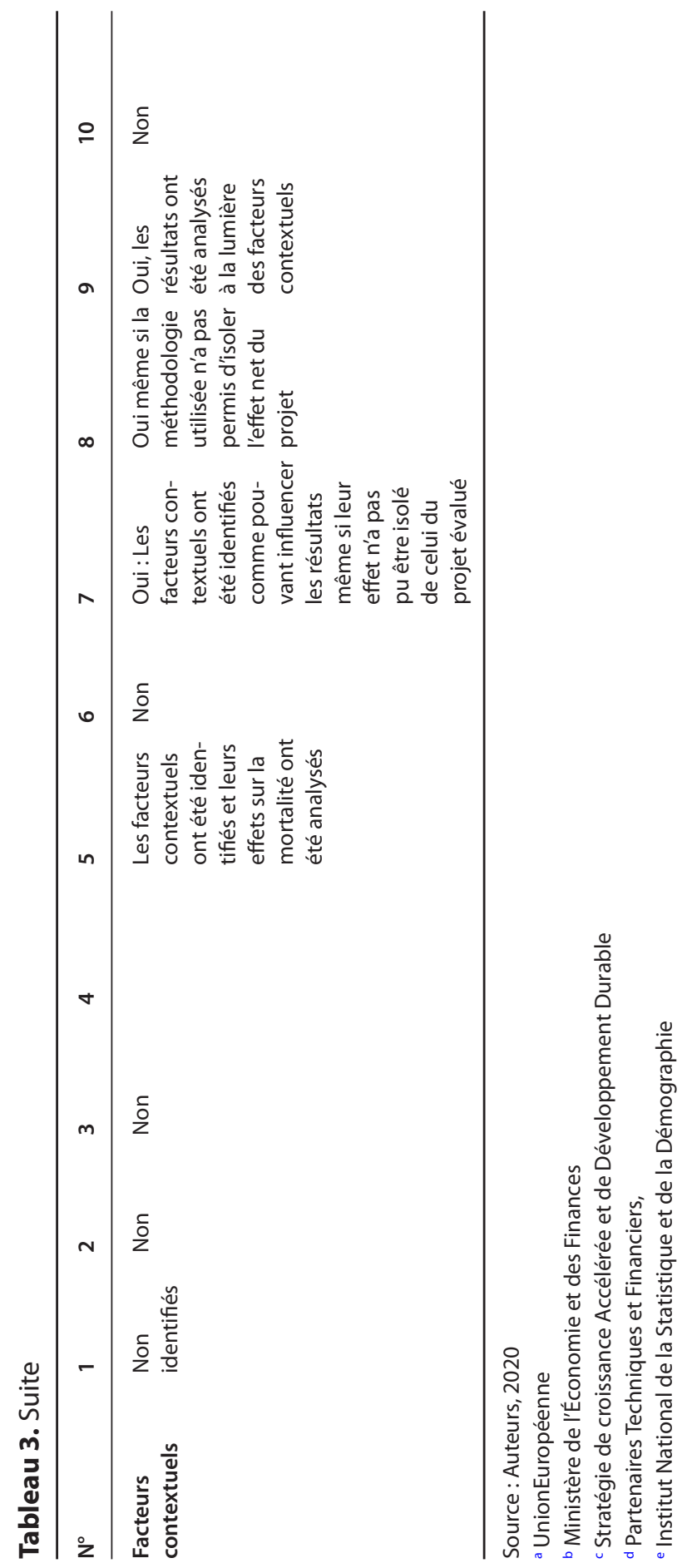


Quant aux méthodes d'évaluation, l'analyse montre que dans la majorité des évaluations de notre échantillon, les méthodes utilisées reposent sur une combinaison d'outils (méthodes quantitative et qualitative). Lutilisation combinée de ces méthodes dans les évaluations est diversifiée et leur usage est le plus souvent indépendant. Pourtant, leur utilisation combinée dans une évaluation devrait être complémentaire pour une meilleure compréhension des résultats; la dimension qualitative devant idéalement servir à apprécier les données quantitatives (Migaud, 2013; Ridde et coll., 2016) ou la méthode quantitative devant permettre de généraliser les résultats qualitatifs (Ridde et coll., 2016).

Par ailleurs, le choix des méthodes (quantitatives comme qualitatives) utilisées n'est pratiquement pas justifié. Dans les rapports utilisant une méthode quasi expérimentale, bien que le choix de l'échantillon soit justifié, il n’en est pas de même pour sa représentativité. Même si les biais en lien avec la stratégie d'analyse sont discutés et les facteurs contextuels identifiés dans quelques rapports, aucun d'entre eux n'a pu isoler l'effet net de l'intervention évaluée. Par ailleurs, dans la plupart des évaluations de notre échantillon, les facteurs contextuels n'ont pas été identifiés. Pourtant, une méta-analyse a montré que les facteurs contextuels qui agissent sur la mise en œuvre des interventions réduisent de moitié l'efficacité théorique de ces interventions (Durlak et DuPre, 2008). Ainsi, la prise en compte de ces facteurs est importante pour évaluer l'effet dû au programme.

Au regard de la nature purement descriptive de cette étude qui s'est focalisée uniquement sur les rapports dévaluation, une analyse approfondie de certains aspects n'a pas pu être faite, notamment en ce qui concerne la démarche évaluative et l'utilisation réservée à ces évaluations. Ainsi, la limite principale de cette étude est liée au fait que nous n'avons pas mené des entretiens avec les différentes parties prenantes, notamment avec les évaluateurs et les commanditaires. Ces entretiens nous auraient permis de collecter les documents connexes aux évaluations (notamment les protocoles dévaluation, les lettres de missions, etc.) qui auraient pu nous aider à mieux comprendre tout le processus d'évaluation et l'utilisation réservée à cesdites évaluations. Ils auraient par ailleurs pu nous permettre d'apprécier les évaluations produites à la lumière des attentes des commanditaires définies généralement dans les TDR ou les protocoles ainsi que l'utilisation qui a été faite de ces évaluations. Pour que les évaluations soient tournées vers la prise de décision, elles doivent être transparentes; la transparence aussi bien dans la conduite de l'évaluation que dans ses résultats qui doivent être rendus publics (Migaud, 2013).

Dagenais et Robert (2012) indiquent que les recommandations issues des rapports d'évaluation ne sont toujours pas utilisées dans les prises de décision. Une équipe d'évaluateurs témoignent leur expérience vécue dans un pays de l'Afrique de l'Ouest au moment de la restitution des résultats. Leurs résultats ont été remis en cause par des participants qui n’approuvaient pas la méthodologie utilisée ou mettaient en doute la compréhension des questions par les enquêtés, ce qui a conduit à la remise en cause des recommandations issues de lévaluation (Kouanda, 2016). Par ailleurs, une étude diagnostique des capacités en évaluation au Burkina Faso, s'appuyant sur un cadre d'analyse des systèmes d'évaluation existants dans quatre pays d'Afrique subsaharienne (Ouganda, Afrique du Sud, 
Bénin et Sénégal), a révélé une faible utilisation des évaluations dans l'ensemble (MINEFID/DEP, 2017). Le même constat est fait au niveau du Burkina Faso. Qu'en est-il pour le cas particulier du secteur de la santé? Un questionnement qui nécessite des entretiens avec les différentes parties prenantes pour fournir des éléments de réponses. Le projet de recherche qui cadre la présente étude va tenter de répondre à ce questionnement. L'analyse seule des rapports d'évaluation, réalisée ici, n’a pas pu explorer la question des utilisateurs de ces évaluations.

\section{CONCLUSION}

Dans cet article, nous fournissons une analyse exploratoire des pratiques évaluatives dans le domaine de la santé au Burkina Faso depuis 2011. Notre stratégie a consisté en une analyse descriptive d'un échantillon raisonné de dix évaluations, menée sur la base d'une grille d’analyse définie au préalable pour identifier les caractéristiques desdites évaluations. L'examen de ces évaluations montre quau Burkina Faso, les pratiques évaluatives dans le secteur de la santé sont assez diversifiées. Cela peut s'expliquer par le fait qu'il n'existe pas jusquà présent de politique évaluative au Ministère de la santé. Ainsi, la décision dévaluer ou non une intervention reste aléatoire et très souvent guidée par les bailleurs de fonds. Les évaluations sont alors faites par projet, ce qui risque de handicaper la capitalisation des résultats.

Nos résultats montrent que les rapports d'évaluation en santé produits ne respectent pas, pour la plupart, les procédures et les normes internationales en matière d'évaluation, notamment en ce qui concerne la définition des questions évaluatives et des critères d'évaluation. Très peu d'évaluations se réfèrent à ces éléments. En fait, les rapports ne fournissent pas assez d'informations pour analyser les liens de causalité entre l'intervention étudiée et ses résultats. Ainsi, ces liens sont parfois établis sans prendre en compte les biais en lien avec les stratégies d'analyse et sans discuter de leur conséquence sur la validité des analyses. Par ailleurs, aucune évaluation n'a pu dissocier l'effet des facteurs contextuels de celui de l'intervention évaluée ce qui a nui à la capacité de généraliser les constats faits.

Nos résultats impliquent que beaucoup d'efforts restent à faire en matière de pratique évaluative dans le secteur de la santé au Burkina Faso. Les décideurs politiques et les planificateurs de programmes et projets de santé dans le pays ont tout intérêt à développer une culture évaluative des politiques, projets et programmes de santé en vue d'une meilleure capitalisation des résultats dans les prises de décision.

\section{BIBLIOGRAPHIE}

Assemblée nationale du Burkina Faso : Commission d'enquête parlementaire sur le système de santé. (2017). Rapport de synthèse de l'enquête parlementaire sur le système de santé au Burkina Faso 2006-2016. Consulté au https://www.assembleenationale.bf/IMG/ pdf/rapport_synthese_amende_vf.pdf

Comité d’aide au développement (CAD). (1991). Principes pour l'évaluation de l'aide au développement. OCDE/GD(91)208. Consulté au http://www.oecd.org/officialdocuments/ publicdisplaydocumentpdf $/$ cote $=\mathrm{OCDE} / \mathrm{GD}(91) 208 \&$ docLanguage $=\mathrm{Fr}$ 
Conseil scientifique de l'évaluation. (1996). Le petit guide de l’évaluation. Paris: La Documentation française.

Cooksy, L. J. et Caracelli, V. J. (2009). Meta-evaluation in practice. Journal of Multidisciplinary Evaluation, 6(11), 1-15.

Coopération internationale et développement EuropeAid. (2016). Evaluation de l'Appui Budgétaire au Burkina Faso (2009-2014). Volume 1 et 2.

Dagenais, C. et Robert, É. (éds.). (2012). Le transfert des connaissances dans le domaine social. Montréal: Presses de l'Université de Montréal.

Daigneault, P. M. (2010). L’examen de la qualité des évaluations fédérales : une métaévaluation réussie. Revue canadienne d’évaluation de programme, 23(2), 191-224.

De Allegri, M., Lohmann, J. et Schleicher, M. (2018). Results-Based Financing for Health Impact Evaluation in Burkina Faso. Results report.

Demarteau, M. (1998). Analyse des pratiques dévaluation de programmes d'éducation pour la santé (thèse de doctorat). Université de Liège, Liège. Consulté au https://orbi.uliege. be/bitstream/2268/78525/1/ELE\%20EVA-PROG\%20R-3774.pdf

Dixey, R. et Njai, M. (2013). The call to action: Health promotion in the Gambia-closing the implementation gap? Global Health Promotion, 20(2), 5-12. https://doi.org/10.1177/ 1757975913486682. Medline:23797935

Dubois, J. M. (2015). La place de l'évaluateur au sein des expérimentations sociales. Agora débats/jeunesses, 69(1), 115-127. https://doi.org/10.3917/agora.069.0115

Durlak, J. A. et DuPre, E. P. (2008). Implementation matters: A review of research on the influence of implementation on program outcomes and the factors affecting implementation. American journal of community psychology, 41(3), 327-350.

Groupe des Nations Unies pour l'évaluation. (2016). Normes et règles d'évaluation. New York: UNEG. Consulté au http://www.unevaluation.org/2016-Norms-and-Standards

Khan, M. S., Guan, B. Y., Audimulam, J., Liceras, F. C., Coker, R. J. et Yoong, J. (2016). Economic interventions to improve population health: A scoping study of systematic reviews. BMC Public Health, 16(1), 528. https://doi.org/10.1186/s12889-016-3119-5. Medline:27386860

Konkobo, T. (2013). Évaluation de projets/programmes dans les pays en voie de développement : cas de quatre projets au Burkina Faso. Sociologie. Université Toulouse le Mirail-Toulouse II. Consulté au https://tel.archives-ouvertes.fr/tel-00976828

Kouanda, S. (2016). Enjeux éthiques et défis méthodologiques de lévaluation en santé en Afrique. Dans Ridde, V., Kouanda, S. et Kobiané, J. F. (éds.), Pratiques et méthodes d'évaluation en Afrique (pp. 97-109). Paris: L'Harmattan.

KPMG et Quadrant conseil. (2017). Evaluation de la démarche globale dévaluation des politiques publiques menée dans le cadre de la modernisation de l'action publique. Consulté au https://www.modernisation.gouv.fr/sites/default/files/fichiers-attaches/ evaluation_epp-map_rapport.pdf

Lavigne-Delville, P. (2011). Vers une socio-anthropologie des interventions de développement comme action publique (Thèse de doctorat). Consulté au https://tel.archives-ouvertes. $\mathrm{fr} / \mathrm{tel}-00683177$ 
Lavigne-Delville, P. (2016). L'évaluation experte des projets de développement : entre instrumentalisation et production d'une analyse partagée. Dans Ridde, V., Kouanda, S. et Kobiané, J. F. (éds.), Pratiques et méthodes d’évaluation en Afrique (pp. 111-146). Paris: L’Harmattan.

Manirambona, R. et Kaboré, S. (2017). Evaluation du projet « Renforcement de l'intégration de la prise en charge de la malnutrition aiguë globale dans le système de santé de la région du Sahel ».

Migaud, D. (2013). Les cinq défis de lévaluation. Revue française d'administration publique, 4(148), 849-858. https://doi.org/10.3917/rfap.148.0849

Mimche, H. (2016). Le recours aux approches qualitatives dans le cadre des évaluations de programme de santé en Afrique. Dans Ridde, V., Kouanda, S. et Kobiané, J. F. (éds.), Pratiques et méthodes dévaluation en Afrique (pp. 223-243). Paris: L'Harmattan.

Ministère de l'Economie, des Finances et du Développement, Direction de l'Economie et de la Planification. (2017) Rapport de l'Étude Diagnostique des Capacités Évaluatives au Burkina Faso.

Ministère de la santé. (2011). Plan national de développement sanitaire 2011-2020. Consulté au https://www.uhc2030.org/fileadmin/uploads/ihp/Documents/Country_Pages/ Burkina_Faso/Burkina_Faso_National_Health_Strategy_2011-2020_French.pdf

Morra, L. G. I. et Rist, R. (2009). The road to results: Designing and conducting effective development evaluations (English). Washington, DC: World Bank. Consulté au http:// documents.worldbank.org/curated/en/400101468169742262/The-road-to-resultsdesigning-and-conducting-effective-development-evaluations

Munos, M., Guiella, G., Roberton, T., Maïga, A., Tiendrebeogo, A., Tam, Y., ... et Baya, B. (2016). Independent evaluation of the rapid scale-up program to reduce under-five mortality in Burkina Faso. The American Journal of Tropical Medicine and Hygiene, 94(3), 584-595.

Organisation de coopération et de développement économiques (OCDE). (2002). Glossary of key terms in evaluation and results-based management, Development Assistance Committee (DAC). Paris, France. Consulté au http://www.oecd.org/dataoecd/29/21/ 2754804.pdf

Olivier de Sardan, J. P. (2011). Promouvoir la recherche face à la consultance. Autour de l'expérience du lasdel (Niger-Bénin). Cahiers d'études africaines, 51(202-203), 511-528. https://doi.org/10.4000/etudesafricaines.16759

Ramaswamy, R., Kallam, B., Kopic, D., Pujic, B. et Owen, M. D. (2016). Global health partnerships: Building multi-national collaborations to achieve lasting improvements in maternal and neonatal health. Globalization and Health, 12(1), 22. https://doi. org/10.1186/s12992-016-0159-7. Medline:27206731

Réseau du CAD de l'OCDE sur l'évaluation du développement. (2019). Des meilleurs critères pour des meilleures évaluations. Définitions adaptées et principes d'utilisation. Consulté au https://www.oecd.org/fr/cad/evaluation/criteres-adaptees-evaluationdec-2019.pdf

Ridde, V. et Dagenais, C. (2012). Approches et pratiques en évaluation de programmes. Montréal: Les Presses de l'Université de Montréal. Consulté au http://books.openedition. org/pum/5965. ISBN: 9782821898004. https://doi.org/10.4000/books.pum.5965 
Ridde, V., Kouanda, S. et Kobiané, J. F. (2016). Pratiques et méthodes d’évaluation en Afrique. Paris: L'Harmattan.

Scriven, M. (2011). Evaluating evaluations: A meta-evaluation checklist. Consulté au http:// michaelscriven.info/images/EVALUATING_EVALUATIONS_8.16.11.pdf.

Service de l'Évaluation spéciale. (2013). Méta-évaluation des programmes des acteurs non gouvernementaux. Bruxelles: SPF Affaires étrangères, Commerce extérieur et Coopération au développement.

Stufflebeam, D. L. (2001). The metaevaluation imperative. American Journal of Evaluation, 22(2), 183-209. https://doi.org/10.1016/s1098-2140(01)00127-8

Widmer, T., Landert, C. et Bachman, N. (2000). Standards dévaluation de la Société suisse d'évaluation (Standards SEVAL). Consulté au https://www.hes-so.ch/data/documents/ SEVAL-Standards-FR-7089.pdf

\section{PRÉSENTATION D'AUTEURS}

Wamadini dite Minata Souratié est titulaire d'un Diplôme d'Etudes Approfondies en Economie Appliquée du Programme de Troisième Cycle Inter-Universitaire et d'un Doctorat en sciences économiques, option Economie Appliquée à l'Université Ouaga II/Burkina Faso. Elle est enseignante-assistante à l'Université de Dédougou (Burkina Faso) et chercheure associée au Laboratoire d'Analyse Quantitative Appliquée au Développement-Sahel (LAQAD-S) /Université Ouaga II. Elle a participé à plusieurs colloques nationaux et internationaux sur diverses thématiques notamment les évaluations des politiques de santé, les inégalités de genre, marché du travail et développement. Ses recherches portent sur la demande de santé, les évaluations des politiques de santé, l'accès des femmes aux ressources productives, le genre et changement climatique, léducation et contrôle des naissances, léducation scolaire et utilisation de la contraception, l'éducation de la petite enfance et développement de l'enfant.

Olivier G. Sossa est détenteur d'un doctorat en santé publique avec une spécialisation en organisation des soins de santé de l'Université de Montréal et d'un DESS en éthique appliquée au domaine de la santé et des services sociaux (Université Laval-Québec).

Ses intérêts professionnels portent sur l'évaluation de programme, l'analyse de politiques publiques, la gestion du changement dans les organisations de la santé et l'intégration de l'éthique dans la prise de décision et l'élaboration de politiques publiques. Il a publié plusieurs articles dans ces domaines particulièrement sur l'implantation d'une culture d'évaluation, la gestion et l'institutionnalisation d'un changement ainsi que la responsabilité populationnelle pour accroitre l'impact des interventions en santé.

Élisabeth Paul est titulaire d'un doctorat en Sciences de Gestion de l'Université de Liège (2006). Elle combine une carrière académique et sur le terrain dans le domaine de l'aide au développement et de la santé mondiale, avec à son actif plus de 80 missions d'appui technique, d'évaluation ou de recherche, principalement en Afrique de l'Ouest. Elle est actuellement chargée de cours et directrice du Centre de Recherche (CR3) «Politiques et systèmes de santé - santé internationale » de l'Ecole de santé publique (ESP) de l'Université libre de Bruxelles (ULB). Elle est aussi consultante indépendante. Ses domaines d'expertise sont les systèmes et politiques de santé mondiale, le financement basé sur la performance, la gestion de l'aide internationale (y compris l'appui budgétaire) et les finances publiques. 


\section{ANNEXE 1}

\section{Liste des rapports d'évaluation inclus dans notre échantillon}

1. Ministère de la santé. (2013). Rapport de la revue à mi-parcours du plan stratégique de lutte contre le paludisme 2011-2015. 81p.

2. AUREC Afrique-BF. (2013). Évaluation des capacités de gestion du programme national de lutte contre le paludisme. 51p.

3. Programme National de Lutte contre le Paludisme et consultant. (2016). Rapport de la revue de performances du programme national de lutte contre le paludisme. 64p.

4. Coopération internationale et développement EuropeAid. (2016). Evaluation de l'Appui Budgétaire au Burkina Faso (2009-2014). Volume 1. $125 \mathrm{p}$ et volume $2.309 \mathrm{p}$.

5. Munos, M., Guiella, G., Roberton, T., Maïga, A., Tiendrebeogo, A., Tam, Y., ... et Baya, B. (2016). Independent evaluation of the rapid scale-up program to reduce under-five mortality in Burkina Faso. The American Journal of Tropical Medicine and Hygiene, 94(3), 584-595.

6. Programme national de lutte contre la tuberculose. (2017). Rapport de la revue du programme national de lutte contre la tuberculose du Burkina Faso. 155p.

7. Manirambona, R. et Kaboré, S. (2017). Evaluation du projet « Renforcement de l'intégration de la prise en charge de la malnutrition aiguë globale dans le système de santé de la région du Sahel ».76p.

8. De Allegri, M., Lohmann, J. et Schleicher, M. (2018). Results-based financing for health impact evaluation in Burkina Faso. Results report. 103p.

9. Nguyen, H. T., Zombré, D., Ridde, V. et De Allegri, M. (2018). The impact of reducing and eliminating user fees on facility-based delivery: A controlled interrupted time series in Burkina Faso. Health Policy and Planning, 33(8), 948-956.

10. Programme national de lutte contre le paludisme. (2019). Rapport de la revue à mi-parcours de la performance du programme de lutte contre le paludisme. 